\title{
Binary Labelings for Plane Quadrangulations and their Relatives
}

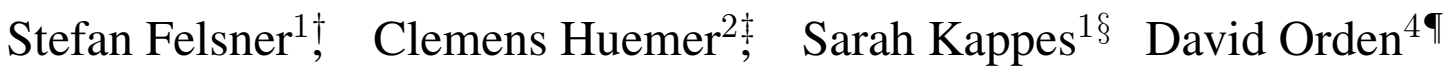 \\ ${ }^{1}$ Institut für Mathematik, Technische Universität Berlin. \{felsner, kappes\} @math.tu-berlin. de \\ ${ }^{2}$ Departament de Matemàtica Aplicada IV, Universitat Politècnica de Catalunya. clemens. huemer@upc. edu \\ ${ }^{3}$ Departamento de Matemáticas, Universidad de Alcalá. david. orden@uah.es
}

received 22 $2^{\text {nd }}$ April 2008, revised $3^{\text {rd }}$ September 2009, accepted $10^{\text {th }}$ July 2010.

\begin{abstract}
Motivated by the bijection between Schnyder labelings of a plane triangulation and partitions of its inner edges into three trees, we look for binary labelings for quadrangulations (whose edges can be partitioned into two trees). Our labeling resembles many of the properties of Schnyder's one for triangulations: Apart from being in bijection with tree decompositions, paths in these trees allow to define the regions of a vertex such that counting faces in them yields an algorithm for embedding the quadrangulation, in this case on a 2-book. Furthermore, as Schnyder labelings have been extended to 3 -connected plane graphs, we are able to extend our labeling from quadrangulations to a larger class of 2-connected bipartite graphs.
\end{abstract}

Keywords: Schnyder labeling, quadrangulation, book embedding, pseudo-triangulation, Laman graph

\section{Introduction}

Schnyder labelings are by now a classical tool to deal with planar graphs. A Schnyder labeling is a special labeling of the angles of a plane graph with three colors. Schnyder [21] introduced this concept for triangulations, i.e., maximal (in the number of edges) planar graphs. He showed that these angle labelings are in bijection with Schnyder woods, i.e., special partitions of the inner edges of the triangulation into three trees. A main application of Schnyder woods are straight-line embeddings of triangulations on small grids. Felsner [5] generalized the concepts of Schnyder labelings and Schnyder woods to the larger class of 3-connected plane graphs. Again, there are applications in graph drawing [3].

Plane quadrangulations are plane graphs all whose faces have degree four. They are also called maximal bipartite planar graphs. The present work is motivated by the fact that quadrangulations admit a decomposition of the edge set into two trees. Our aim is to look for a closer resemblance of Schnyder structures in these cases. In particular we study angle labelings with two colors.

\footnotetext{
${ }^{\dagger}$ Partially supported by grant DFG FE 340/7-1

¥Partially supported by projects MEC MTM2009-07242 and Gen. Cat. DGR 2009SGR1040.

§Supported by the DFG trough the int. RTG 'Comb., Geom. and Comp.' (No. GRK 588/2).

\ Research partially supported by grant MTM2008-04699-C03-02. 
In Section 2 we define strong labelings. A graph admitting a strong labeling has to be a plane quadrangulation. We show that strong labelings indeed resemble many properties of Schnyder labelings:

- Strong labelings induce a partition of the quadrangulation into two oriented trees with Schnyder-like properties, see Subsection 2.1. For the existence of a 2-tree decomposition of a plane quadrangulation there are many references, e.g. [1, 8, 9, 10, 13, 15, 17, 18]. The tree decomposition induced by the strong labeling has the nice property that at each vertex the two trees are "separated", i.e., around a vertex the edges of each tree appear consecutively. Such separating tree decompositions have been previously studied in [8]. In fact, strong labelings and separating decompositions are in bijection. Further combinatorial objects related to strong labelings and separating decompositions have recently been studied in [7].

- Strong labelings also allow to obtain an embedding of a quadrangulation on a 2-book, i.e., a mapping of the nodes to a line and a non-crossing embedding of the edges in the half-planes separated by that line. In our case each halfplane contains the edges of one of the two trees. Let $v_{1}, \ldots, v_{n}$ be the nodes ordered along the line. Then the trees on each page are alternating, i.e., there are no two edges $v_{i} v_{j}$ and $v_{j} v_{k}$ with $i<j<k$. Book embeddings of graphs are well studied and have several applications; see e.g. [4]. For the particular case of quadrangulations, the existence of a 2-book embedding with a tree on each page was shown in [9]. Our Schnyder-like technique allows to obtain the alternating property. Non-crossing alternating trees were studied and counted in [11]. They have also appeared as one-dimensional analogs of pseudo-triangulations [19].

In Section 3 we define weak labelings for plane graphs. Weak labelings contain strong labelings as a subclass. Every weak labeling induces a 2-coloring and a 2-orientation of the $2 n-4$ edges ( $n$ being the number of vertices). We show that weak labelings are indeed in bijection with a pair of 2-orientations, one for the graph and another for an appropriately defined dual. This allows the characterization and efficient recognition of graphs admitting a weak labeling. We remark that a slight variant of weak labelings also exists for the class of Laman graphs [12].

In Section 4 we generalize the notion of strong labeling from quadrangulations to a larger class of bipartite graphs. The generalized strong labelings still yield a pair of trees. This is similar to the generalization of Schnyder structures in [5]. The class of bipartite graphs admitting a generalized strong labeling is characterized in Subsection 4.2

\section{Strong labelings}

Definition 2.1 Let $G$ be a quadrangulation with color classes of black and white vertices and two distinguished black vertices $s_{0}$ and $s_{1}$ on the outer face. A strong labeling of $G$ is a mapping of the angles of $G$ to $\{0,1\}$ which satisfies:

(G0) Special vertices: All angles incident to $s_{i}$ are labeled $i$.

(G1) Vertex rule: For each vertex $v \notin\left\{s_{0}, s_{1}\right\}$, the incident labels form a non-empty interval of $1 s$ and a non-empty interval of $0 s$.

(G2) Edge rule: For each edge, the incident labels coincide at one endpoint and differ at the other.

(G3) Face rule: The labels in each face are 0011 cyclically. Reading the labels of the outer face in clockwise order starting at $s_{0}$, they are also 0011. 
Observation 2.2 A strong labeling induces both a 2-coloring and a 2-orientation of the edges: Every edge is colored according to its endpoint with the two coincident labels and oriented towards that endpoint. Moreover, the vertex rule implies that every vertex except $s_{0}, s_{1}$ has outdegree two; such an orientation will be called a 2-orientation. See Figure 1

In the figures, and sometimes in the text we will identify color 0 with gray and color 1 with black.
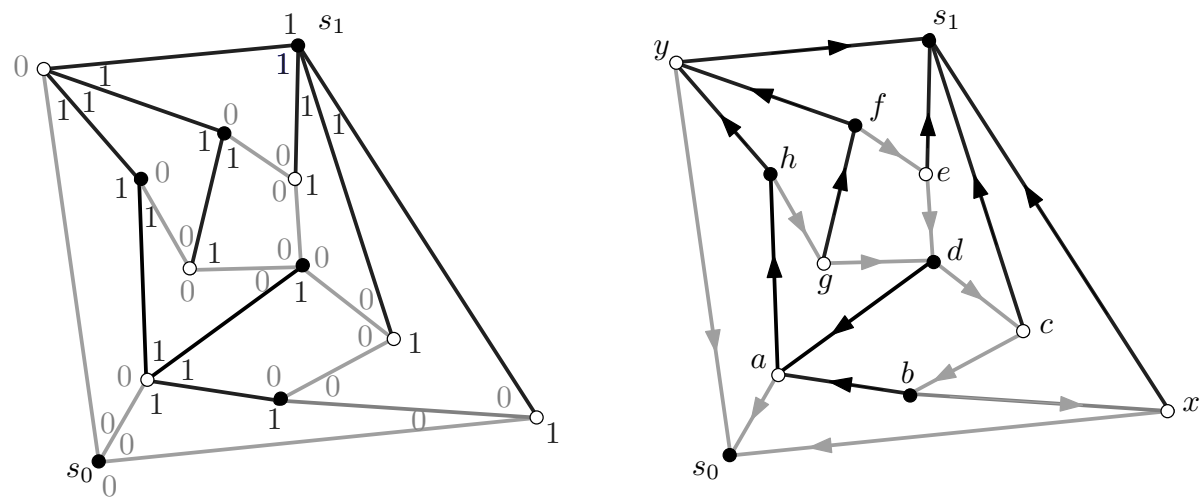

Fig. 1: A strong labeling for a quadrangulation (left) and the induced 2-coloring and orientation of the edges (right).

Lemma 2.3 (Walking rule) In a strong labeling of a quadrangulation the following is true: Walking along an interior face in clockwise order, the labels change precisely when moving from a black to a white vertex.

Proof. Let $F$ and $F^{\prime}$ be two faces sharing an edge $e$. Suppose that $F$ obeys the walking rule. If the clockwise walk in $F$ sees a change of labels along $e$, then this walk traverses $e$ from the black to the white vertex, which determines the partition into black and white for all vertices on $F$ and $F^{\prime}$. The edge rule implies that the two labels on the other side of $e$ are the same. This observation together with the face rule for $F^{\prime}$ yields the validity of the walking rule for $F^{\prime}$. The other possibility, when the clockwise walk in $F$ sees the same label on both endpoints of $e$, is similar: The walking rule determines the black/white partition, the edge rule implies two different labels in $F^{\prime}$ and the face rule enforces the walking rule for $F^{\prime}$.

From the definition of labels at the outer face we obtain the validity of the walking rule for the bounded face $F_{0}$ which is incident to the edge containing $s_{0}$ and having two labels 0 on the outer face. Any face $F$ can be connected to $F_{0}$ with a dual path avoiding the outer face. The above reasoning allows to transfer the validity of the walking rule along this path to $F$.

The following strong edge rule is an immediate consequence of the edge rule together with the walking rule. Actually, the walking rule also follows from the strong edge rule, i.e., the two rules are equivalent.

Lemma 2.4 (Strong edge rule) In a strong labeling of a quadrangulation the following is true: For each edge, the incident labels coincide at one endpoint and differ at the other. Moreover if the latter is a white (respectively black) vertex, the right (respectively left) side of the edge, oriented as in Observation 2.2. has coincident labels. See Figure 2. 


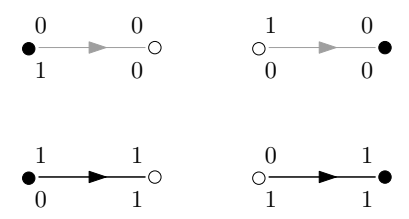

Fig. 2: Edge types complying with the strong edge rule.

Yet another useful property of strong labelings is given with the next lemma, whose proof is immediate from the strong edge rule. Observe that, as in the previous lemma, the rule is "white-right", "black-left".

Lemma 2.5 (Turning rule) In a strong labeling of a quadrangulation the following is true: If $v$ is a white (respectively black) vertex and $u v$ an incoming edge, then the outgoing edge at $v$ with the same color as $u v$ is the next outgoing edge to the right (respectively left) of uv. See Figure 3
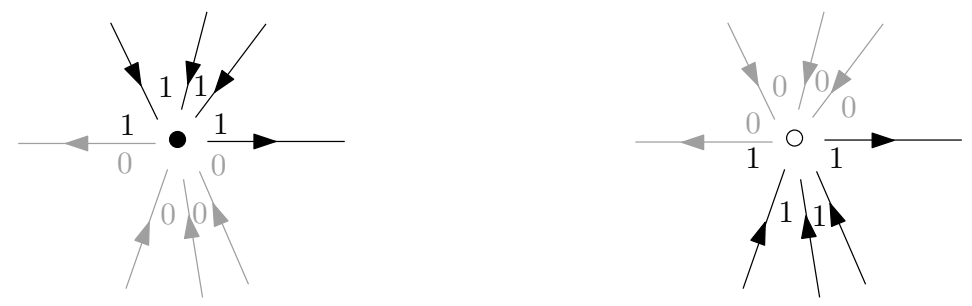

Fig. 3: Illustrating the turning rule.

Before further studying strong labelings of quadrangulations we prove that every quadrangulation has such a labeling.

Theorem 2.6 Every quadrangulation admits a strong labeling.

Proof. We use induction on the number of vertices $n$ of a quadrangulation $Q$. If $n=4$ then a strong labeling exists, as shown in Figure 4 (left). For the induction step we distinguish two cases.

For the first case, assume that $Q$ contains an interior vertex $v$ of degree two. Removal of $v$ and its two incident edges yields a quadrangulation $Q^{\prime}$ which, by induction, admits a strong labeling. Reinsertion of $v$ and its incident edges into $Q^{\prime}$ can be done in a unique way such that the rules of strong labelings are maintained. One of the possible cases is shown in Figure4 (right).

For the second case, assume that $Q$ contains no interior vertex of degree two. We say that a face $q$ incident to $s_{0}$ is contractible if it does not contain the other special vertex $s_{1}$. The contraction of $q=$ $\left\{e^{\prime}, e, f, f^{\prime}\right\}$, where $\left\{e^{\prime}, e, f, f^{\prime}\right\}$ are the edges of $q$ in clockwise order starting at $s_{0}$ and $p$ is the vertex opposite to $s_{0}$, identifies $e$ with $e^{\prime}, f$ with $f^{\prime}$ and $p$ with $s_{0}$. This can be interpreted as a continuous movement of $p$ and its incident edges to $s_{0}$, see Figure 5 .

A contraction makes a problem when it creates a double edge. This happens when $p$ and $s_{0}$ have three common neighbors, in this case the contraction is illegal. To find a legally contractible face $q$ we proceed as follows: let $p_{0}=p$ and let $q_{0}$ be any face incident to the black vertices $s_{0}$ and $p_{0}$. Let $R_{0}$ be the 

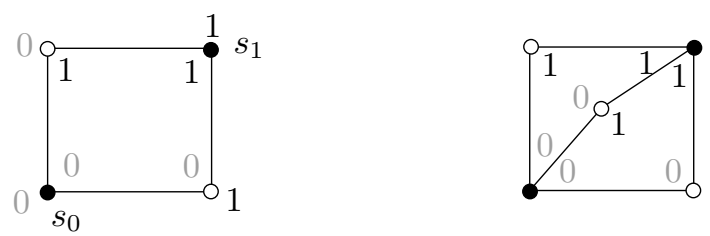

Fig. 4: The basis of the induction and inserting a vertex of degree two.

region obtained as union of all four-cycles through $s_{0}$ and $p_{0}$ together with their interiors. If $R_{0}=q_{0}$, then contracting $q_{0}$ is legal. Otherwise there is a white vertex in the strict interior of $R_{0}$. Since this white vertex has degree $\geq 3$ there is a black vertex $p_{1}$ such that $p_{1}$ is on a common face $q_{1}$ with $s_{0}$ and $R_{1} \subsetneq R_{0}$, where $R_{1}$ is the region defined by the four-cycles through $p_{1}$ and $s_{0}$. Iterate this until a region $R_{k}$ consists of a single face $q_{k}$, this has to happen by finiteness.
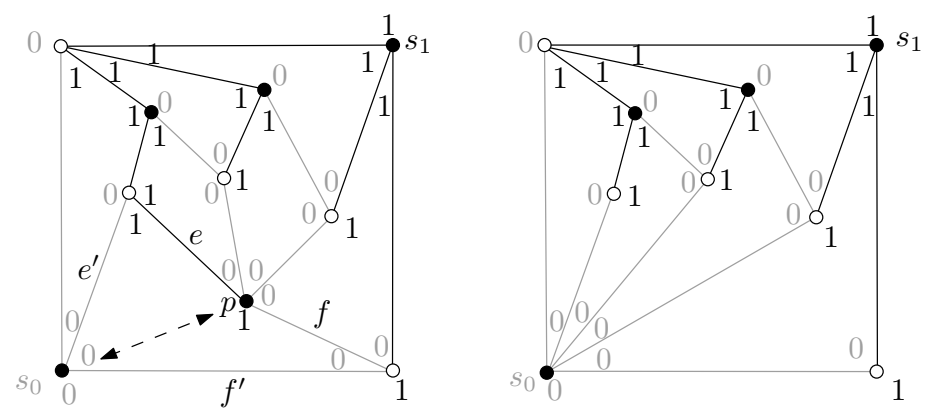

Fig. 5: Contracting a quadrangle to the special vertex $s_{0}$.

The contraction of a face $q$ yields a quadrangulation which by induction admits a strong labeling. Now, reversing the contraction maintains the strong labeling outside of the face $q$ and it only remains to label the angles inside $q$. First, the rule for the special vertex $s_{0}$ requires that the angle at this vertex is labeled 0 . The other labels have to be chosen according to the walking rule. Figure 5 shows an example. The vertex and edge rules at the boundary of the new face are easily verified.

\subsection{Schnyder-like properties for strong labelings}

Consider the coloring and orientation of the edges induced by a strong labeling (c.f. Observation 2.2. For this coloring and orientation we obtain results which are in nice correspondence to those obtained by Schnyder [21] for triangulations and Felsner [5] for 3-connected plane graphs. The orientation and coloring of the edges of a graph induced by a strong labeling have another interesting property: Let $G$ be a quadrangulation with a strong labeling and let $T_{0}$ and $T_{1}$ be the edges of colors 0 and 1 . Since the edges are oriented according to a 2 -orientation, we can define $T_{i}^{-1}$ as the set of edges colored $i$ with their orientation reversed.

Lemma 2.7 Every vertex except $s_{0}, s_{1}$ has outdegree 1 in each of $T_{0}$ and $T_{1}$.

Proof. This follows from the turning rule (Lemma 2.5. 
Lemma 2.8 There is no directed cycle in $T_{0} \cup T_{1}^{-1}$, nor in $T_{1} \cup T_{0}^{-1}$.

Proof. Suppose that there is a cycle in $T_{0} \cup T_{1}^{-1}$. Choose $C$ to be such a cycle with the least number of faces in its interior. Claim 1: There is no vertex in the interior of $C$. Otherwise the black and the gray path leaving the vertex can be used to identify a cycle with less interior faces. Claim 2: $C$ has no chord. Again this follows from the minimality assumption. To complete the proof it can be checked that there is no directed facial cycle in $T_{0} \cup T_{1}^{-1}$.

Corollary $2.9 T_{i}, i \in\{0,1\}$, is a directed tree with sink $s_{i}$ that spans all vertices but $s_{1-i}$.

Proof: From Lemma 2.8 we know that $T_{i}$ has no directed cycle. This and Lemma 2.7 implies that $T_{i}$ is cycle-free. Since $T_{i}$ spans all vertices except $s_{1-i}$ the claim follows.

For each non-special vertex $v \notin\left\{s_{0}, s_{1}\right\}$, we define the $i$-path $P_{i}(v), i \in\{0,1\}$, as the directed path in $T_{i}$ from $v$ to the sink $s_{i}$. We say that two paths cross if they share a vertex $v$ such that the two edges incident to $v$ of one path lie on different sides of the other path.

Observation 2.10 Two paths of the same color cannot cross, because every vertex has outdegree 1 in this color. Two paths of different colors cannot cross, because this would violate the vertex rule.

Lemma 2.11 The paths $P_{i}(v), v \notin\left\{s_{0}, s_{1}\right\}$, are chord-free.

Proof. Let $v=v_{0}, v_{1}, v_{2}, \ldots, v_{k}, s_{i}$ be the sequence of vertices of $P_{i}(v)$. Suppose that $v_{i} v_{j}$ with $i+1<j$ is an edge of the quadrangulation. The edge is not in the tree $T_{i}$, hence, it is of color $1-i$. Lemma 2.8 implies that the orientation is not from $v_{i}$ to $v_{j}$. If $v_{j} v_{i}$ lies to the right of $P_{i}(v)$ we know that $v_{j}$ is black because of the turning rule (Lemma 2.5). The same rule at the white vertex $v_{i}$ implies that the outgoing edge at $v_{i}$ points into the interior of the cycle $v_{i}, v_{i+1}, \ldots, v_{j}, v_{i}$. This implies a crossing between the paths $P_{i}\left(v_{i}\right)$ and $P_{1-i}\left(v_{i}\right)$ which contradicts Observation 2.10 The other case where $v_{j} v_{i}$ lies to the left of $P_{i}(v)$ is essentially symmetric.

Because of Observation 2.10 the paths $P_{0}(v)$ and $P_{1}(v)$ have $v$ as only common vertex. Therefore they split the quadrangulation into two regions which we denote by $R_{0}(v)$ and $R_{1}(v)$, where $R_{i}$ is the region to the right of $P_{i}(v)$ and including both paths.

Lemma 2.12 Let $u, v$ be distinct interior vertices. For $i \in\{0,1\}$, the following implications hold:

(i) $u \in \operatorname{int}\left(R_{i}(v)\right) \Rightarrow R_{i}(u) \subset R_{i}(v)$.

(ii) $u \in P_{i}(v), u \neq v \Rightarrow\left\{\begin{array}{lll}R_{i}(u) \subset R_{i}(v) \quad \text { and } & R_{1-i}(v) \subset R_{1-i}(u) \\ \text { or } & & \\ R_{i}(v) \subset R_{i}(u) & \text { and } \quad & R_{1-i}(u) \subset R_{1-i}(v)\end{array}\right.$.

Proof: If $u \in \operatorname{int}\left(R_{i}(v)\right)$, Observation 2.10 implies that both paths $P_{0}(u), P_{1}(u)$ and the region they enclose are contained in $R_{i}(v)$. If $u \in P_{0}(v), u \neq v$, then $P_{0}(u) \subset P_{0}(v)$ while the first edge of $P_{1}(u)$ points to the interior of either $R_{0}(v)$ (if $u$ is black) or to the interior of $R_{1}(v)$ (if $u$ is white), because of the turning rule. In the first case we obtain $R_{0}(u) \subset R_{0}(v)$ and $R_{1}(u) \supset R_{1}(v)$, in the second case we obtain the reversed inclusions. Similar arguments work if $u \in P_{1}(v), u \neq v$. 


\subsection{Alternating embedding of quadrangulations in a 2-book}

Mimicking the obtention of straight-line embeddings of triangulations on small grids via Schnyder labelings, Lemma 2.12 allows us to obtain 2-book embeddings of quadrangulations such that each of the two pages contains an alternating tree.

For each non-special vertex $v$, let us define $f_{i}(v)$ as the number of faces contained in $R_{i}(v)$. For the two special vertices $s_{0}, s_{1}$, we set $f_{0}\left(s_{0}\right)=f_{1}\left(s_{1}\right)=-1$ and $f_{1}\left(s_{0}\right)=f_{0}\left(s_{1}\right)=n-2$, where $n$ is the number of vertices. As shown in Lemma 2.12 there is an inclusion between the $i$-regions of any two vertices, therefore, the following holds:

Proposition 2.13 For any two vertices $u \neq v$, we have $f_{i}(u) \neq f_{i}(v)$. Equivalently, all possible values of $f_{i}$ from 0 to $n-3$ occur.

All the points $\left(f_{0}(v), f_{1}(v)\right)$ lie equally spaced on the line $f_{0}+f_{1}=f$, where $f$ is the total number of bounded faces (which equals $n-3$ by Euler's formula). For the sake of convenience, we can choose a reference system in which this line is the horizontal axis and the $f_{1}$-values increase from left to right. Given this as spine of the book, we draw the edges of each tree $T_{i}$ on one side. As a convention, we will draw $T_{0}$ gray and above the line, and $T_{1}$ black and below. In Theorem 2.14 we prove that the trees are non-crossing, and hence we get a 2-book embedding for the quadrangulation $Q$ such that each page contains a tree.

In Theorem 2.14 we additionally prove that both trees are alternating, meaning that the tree contains no two edges $v_{i} v_{j}$ and $v_{j} v_{k}$ for $i<j<k$ (where $v_{1}, \ldots, v_{n}$ denotes the vertices in the order they are encountered along the line). This is equivalent to saying that either all neighbors of $v_{j}$ have indices bigger than $j$ or they all have indices smaller than $j$. Figure 6 shows an example for the book embedding.

Non-crossing alternating trees are counted by the Catalan numbers. They came up in research about pseudo-triangulations, where they have been identified as one-dimensional analogs to pseudo-triangulations [19]. In that paper it has been shown that the "flip graph" on alternating trees is the 1-skeleton of the associahedron.

\begin{tabular}{c|cccccccccccc} 
& $a$ & $b$ & $c$ & $d$ & $e$ & $f$ & $g$ & $h$ & $x$ & $y$ & $s_{0}$ & $s_{1}$ \\
\hline$f_{0}$ & 1 & 2 & 8 & 3 & 7 & 6 & 5 & 4 & 9 & 0 & -1 & 10 \\
$f_{1}$ & 8 & 7 & 1 & 6 & 2 & 3 & 4 & 5 & 0 & 9 & 10 & -1
\end{tabular}

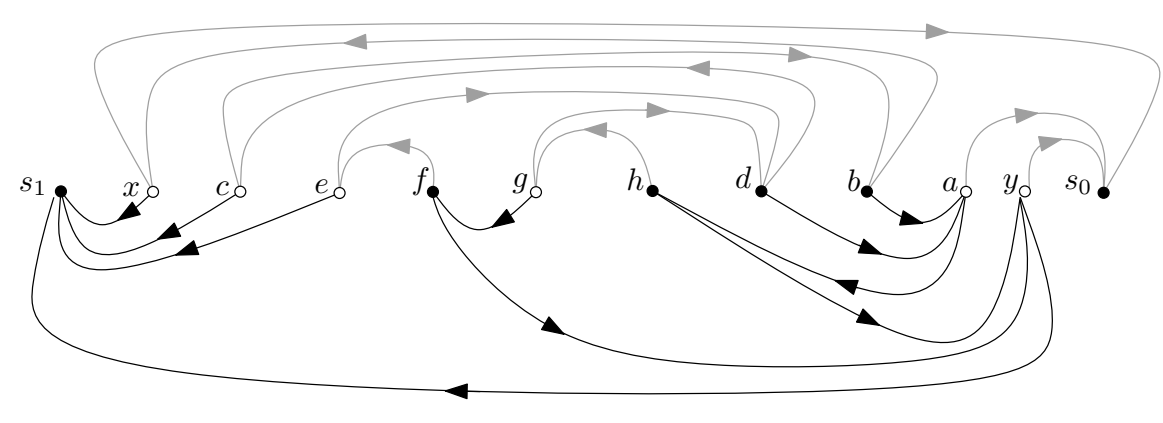

Fig. 6: Embedding on a 2-book the quadrangulation in Figure 1 
Theorem 2.14 Let the vertices of a quadrangulation be placed on a line by the face-counting process, with the trees $T_{0}$ and $T_{1}$ placed on each side of the line. Then $T_{0}$ and $T_{1}$ are non-crossing and alternating.

Proof. We will prove that the gray tree $T_{0}$ cannot have crossings. Let us suppose that there is a crossing in $T_{0}$, i.e., four points $a, b, c, d$ with

$$
f_{0}(a)>f_{0}(b)>f_{0}(c)>f_{0}(d)
$$

and edges $a c, b d$. We focus on the edge $a c$. The two possible configurations are shown in Figure 7 Any other situation would violate either the relations in (1) or the vertex rule (G1).
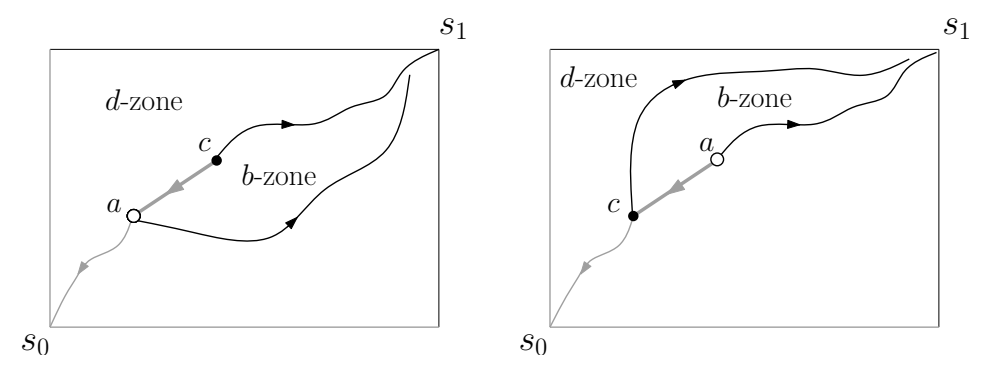

Fig. 7: Possible configurations according to the relations in (1).

Furthermore, from $f_{0}(c)>f_{0}(d)$ and Lemma 2.12, we know that $d \notin \operatorname{int}\left(R_{1}(c)\right)$ and analogously that $b \notin \operatorname{int}\left(R_{0}(c)\right)$. This gives us the feasible zones for points $b$ and $d$, denoted as $b$ - and $d$-zones in Figure 7. Note that in both cases shown in the figure the path $P_{1}(c)$ separates the two zones, hence, the existence of a gray edge $b d$ implies that $b$ or $d$ is on this path. From Lemma 2.11 we know that at most one of them is on the path.

- If $b \in P_{1}(c), d \notin P_{1}(c)$ : In this case the edge $b d$ is to the left of $P_{1}(c)$ and hence the same holds for all the gray edges incident to $b$. Therefore, $R_{0}(b) \subset R_{0}(c)$ which implies $f_{0}(b)<f_{0}(c)$, a contradiction.

- If $b \notin P_{1}(c), d \in P_{1}(c)$ : In this case the edge $b d$ is to the right of $P_{1}(c)$ and hence the same holds for all the gray edges incident to $d$. This leads to the contradiction $f_{0}(d)>f_{0}(c)$.

A similar analysis shows that the black tree $T_{1}$ has no crossings.

We now show that for our choice of coordinates, $T_{0}$ and $T_{1}$ are alternating. We focus on the black tree $T_{1}$, and the case for the gray tree $T_{0}$ is analogous. Vertices $s_{0}$ and $s_{1}$ have all their neighbors on one side, hence they are alternating. For the other two vertices $x$ and $y$ of the external face, all the interior angles are labeled 0 , respectively 1 for $y$, and $x$ is incident to only one edge of $T_{1}$ to $s_{1}$, and $y$ to only one edge of $T_{0}$ to $s_{0}$. This implies that also $x$ and $y$ are alternating. Let us consider an interior black vertex $v_{j}$. The successor $v_{s}$ of $v_{j}$ on the black path $P_{1}\left(v_{j}\right)$ is a white vertex. From the turning rule (Lemma 2.5) it follows that the gray outgoing edge of $v_{s}$ is to the left of $P_{1}\left(v_{j}\right)$, i.e., it points into $R_{0}\left(v_{j}\right)$ which implies $f_{0}\left(v_{s}\right)<f_{0}\left(v_{j}\right)$, equivalently $f_{1}\left(v_{s}\right)>f_{1}\left(v_{j}\right)$ and hence $s>j$. 
Now consider a black edge $v_{p} v_{j}$ which is incoming at $v_{j}$. This edge belongs to the black path $P_{1}\left(v_{p}\right)$. The fact that $v_{j}$ is black and the turning rule implies that the gray outgoing edge of $v_{j}$ points into $R_{1}\left(v_{p}\right)$ which implies $f_{1}\left(v_{p}\right)>f_{1}\left(v_{j}\right)$ and hence $p>j$.

The case where $v_{j}$ is a white vertex is similar, in that case all neighbors in the black tree have indices smaller than $j$.

\subsection{Strong labelings, separating decompositions and 2-orientations}

The following definition was essentially (with reversed orientations) proposed by de Fraysseix and Ossona de Mendez [8].

Definition 2.15 Let $Q$ be a quadrangulation, with vertices of the bipartition properly bicolored as black and white. Let $s_{0}$ and $s_{1}$ be nonadjacent vertices at the outer face. A separating decomposition of $Q$ is a partition of the edges into two directed trees $T_{0}, T_{1}$ with sinks $s_{0}, s_{1}$, such that the incident edges at each vertex but $s_{0}$ and $s_{1}$ are gathered as follows, in clockwise order for black vertices and counterclockwise order for white vertices:

- The incoming edges (if any) from $T_{0}$,

- The outgoing edge from $T_{0}$,

- The incoming edges (if any) from $T_{1}$,

- The outgoing edge from $T_{1}$.

Note that the above condition about the orientations of edges at a vertex is exactly the turning rule (see Figure 3).

Theorem 2.16 Separating decompositions and strong labelings of a quadrangulation are in bijection.

Proof. Let $Q$ be a quadrangulation with a distinguished vertex $s_{0}$ on the outer face. A strong labeling of $Q$ induces a coloring and orientation of the edges. By Corollary 2.9 this yields a partition into trees $T_{0}$ and $T_{1}$ rooted at $s_{0}$ and the opposite vertex of the outer face $s_{1}$. The coloring and orientation of the edges obeys the turning rule (Lemma 2.5). This rule is precisely the condition required for a separating decomposition.

Conversely, let a separating decomposition be given. Given a directed edge $u v$ color both angles incident to $u v$ at $v$ with the color of the edge. The property of a separating decomposition implies that angles with two incident incoming edges get the same label from both edges. Furthermore, the angles at the tail endpoint of an edge being labeled according to the turning rule implies the strong edge rule to be fulfilled. See Figures 2 and 3 It is obvious that the vertex conditions (G0) and (G1) hold for this labeling. All edges conform to the strong edge rule and hence to the edge rule (G2). The strong edge rule also implies the walking rule (Lemma 2.3) which in turn implies the face rule (G3). Together this shows that the implied labeling of angles is a strong labeling.

To enhance the picture we quote the following theorem from [8]. In the statement 'quadrangulation' is again to be understood as a quadrangulation together with a distinguished vertex $s_{0}$ on the outer face.

Theorem 2.17 (De Fraysseix and Ossona de Mendez) Separating decompositions and 2-orientations of a quadrangulation are in bijection.

Corollary 2.18 There is a bijection between 2-orientations and strong labelings of a quadrangulation. 


\subsection{Flips on strong labelings of quadrangulations}

Given a graph $G$ with a 2-orientation, one can reverse any directed cycle and obtain another 2-orientation. Such a local modification of an object in more general terms is often called fip. In Lemma2.19 below we show that for a strong labeling of a quadrangulation, such a flip means that we invert all labels inside the cycle to obtain another strong labeling of the same quadrangulation, see Figure 8
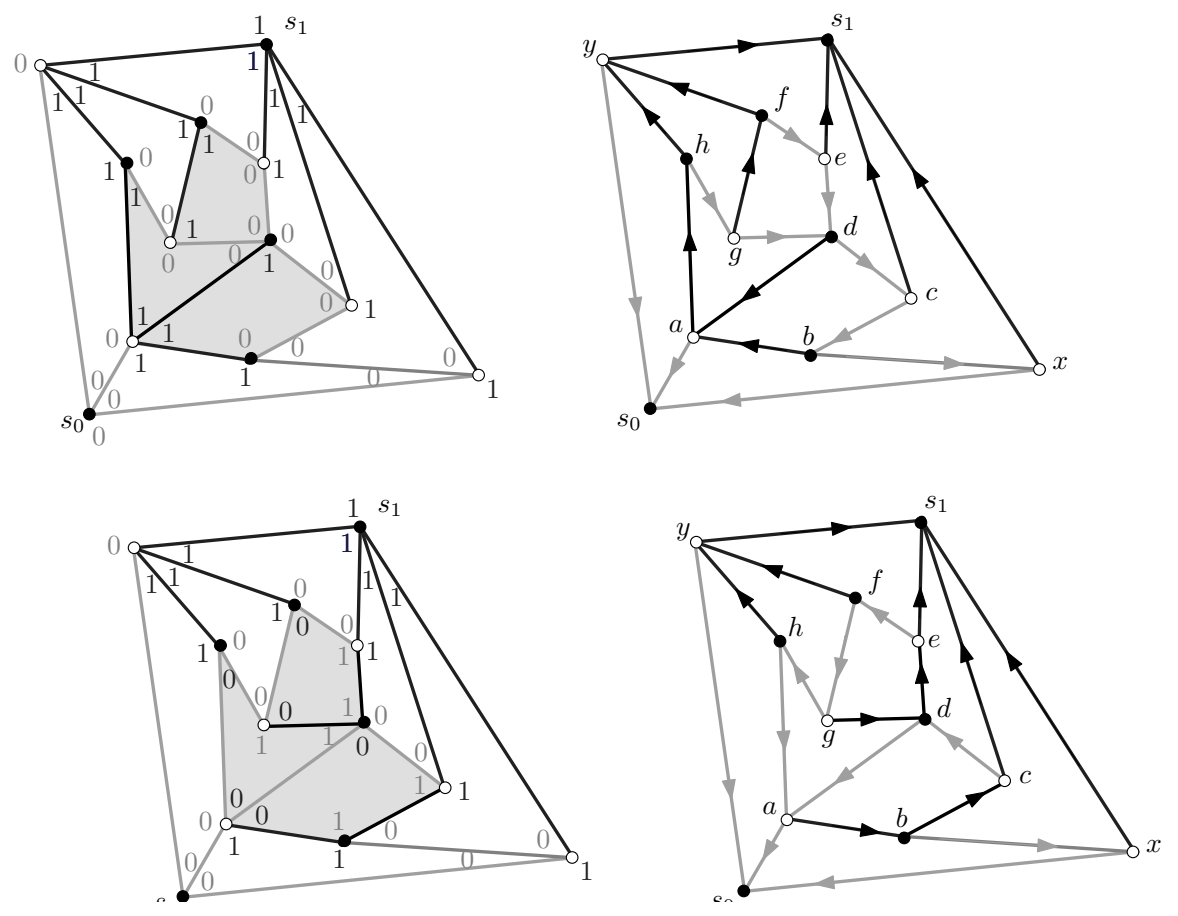

Fig. 8: A flip for a strong labeling of a quadrangulation.

Lemma 2.19 Given a quadrangulation, the reversal of a directed cycle $C$ in a 2-orientation conforms with the complementation of all the labels inside $C$ in the corresponding strong labeling.

Proof: It is obvious that the walking rule and face rule (G3) are maintained for all faces. For the vertices and edges inside or outside the cycle, it is also clear that the vertex rule (G1) and edge rule (G2) are maintained.

Let us consider a vertex $v$ on the boundary of the directed cycle, and let $u v, v w$ be the incident directed edges of the cycle. Before the flip, one of the change of label around $v$ happens at the tail angles of $v w$ and the other change happens either inside or outside the cycle. After the flip, that change happens at the head of $u v$, while the other change keeps its position. This proves the vertex rule (G1).

For an edge on the boundary of the cycle, the two labels that are reversed are either different, in which case they remain different, or the same, in which case they remain the same after the flip. Therefore, the edge rule $(\mathrm{G} 2)$ is satisfied. 
Schnyder woods of triangulations are in bijection to 3-orientations. In this context Brehm [2] has investigated the reversal of directed cycles (flip) for 3-orientations. He proved that the set of 3-orientations forms a distributive lattice. A more general result was obtained by Ossona de Mendez [16] and Felsner [6], they obtained lattice structures on the set of $\alpha$-orientations of a planar graph. A particular instance of the general theorem is that the set of all 2-orientations of a quadrangulation can be enhanced with an ordering which is a distributive lattice. The order relation is the transitive closure generated by $X<X_{C}$ whenever $X$ is a 2-orientation which has a simple directed cycle $C$ which runs clockwise around its interior and $X_{C}$ is obtained by reverting $C$ in $X$. The flip structure on 2-orientations of quadrangulations was also investigated by Nakamoto and Watanabe [15]. A simple consequence of the distributive lattice structure on 2-orientations and the bijection with strong labelings is:

Corollary 2.20 The flip graph of strong labelings is connected.

\section{Weak labelings}

Definition 3.1 Let $G$ be a plane graph with two special vertices $s_{0}$ and $s_{1}$ on the outer face. A weak labeling for $G$ is a mapping from the angles of $G$ to $\{0,1\}$ which satisfies the following conditions:

(G0) Special vertices: All angles incident to $s_{i}$ are labeled $i$.

(G1) Vertex rule: For each vertex $v \notin\left\{s_{0}, s_{1}\right\}$, the incident labels form a non-empty interval of $1 s$ and a non-empty interval of $0 s$.

(G2) Edge rule: For each edge, the incident labels coincide at one endpoint and differ at the other.

$\left(\mathrm{G} 3_{w}\right)$ Weak face rule: For each face (including the outer face), its labels form a non-empty interval of $1 s$ and a non-empty interval of $0 s$.

Observation 3.2 A weak labeling induces both a 2-coloring and a 2-orientation of the edges: Every edge is colored according to its endpoint with the two coincident labels and oriented towards that endpoint. As in the case of strong labelings, such an orientation is a 2-orientation. See Figure 9
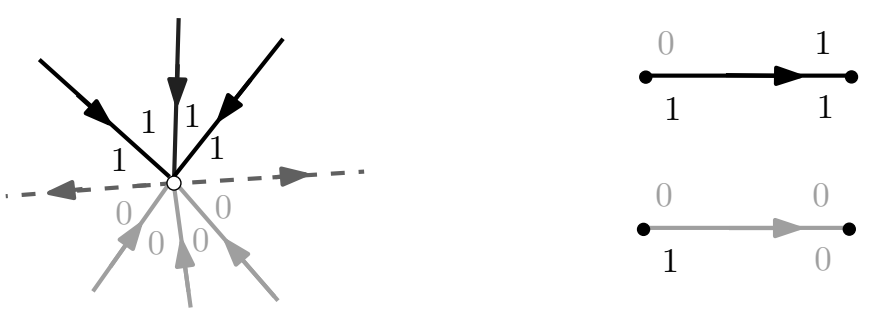

Fig. 9: The orientation induced by a weak labeling, the dashed edges may have either color.

Note that if a graph admits a labeling satisfying (G0), (G1) and (G2), then its edge cardinality is equal to $2 n-4$. A quadrangulation on $n$ vertices has $2 n-4$ edges and indeed quadrangulations admit weak labelings (they even admit a stronger labeling, see Section 2). But weak labelings also exist for some graphs which are not quadrangulations; consider e.g. the graph obtained by inserting into the cycle $C_{6}$ the edges 15 and 24. A more complex example is part of Figure 10. 


\subsection{Graphs admitting a weak labeling}

Observation 3.3 Since the angles of a plane graph $G$ and of the dual $G^{*}$ are in bijection we can interpret a weak labeling $\phi$ of $G$ as a labeling $\phi^{*}$ of the angles of $G^{*}$. The edge rule for $\phi$ is the edge rule for $\phi^{*}$ and the weak face rule for $\phi$ implies the vertex rule for (all!) vertices in the labeling $\phi^{*}$. As in the previous observation, we can argue that $\phi^{*}$ induces an orientation of the edges of $G^{*}$ such that every vertex has outdegree two. One additional property of the orientation induced by $\phi$ can be noted: The special vertices $s_{0}, s_{1}$ divide the edge set on the outer face of $G$ into two arcs $A_{0}$ and $A_{1}$. Each of these arcs contains a change of label. Split the dual vertex corresponding to the outer face of $G$ into two vertices $o_{0}^{*}$ and $o_{1}^{*}$ such that $o_{i}^{*}$ keeps the incidences with the dual edges of $A_{i}$ and let $G_{s}^{*}$ denote the resulting split-dual of $G$. The orientation induced by $\phi$ on $G_{s}^{*}$ has outdegree one at $o_{0}^{*}$ and $o_{1}^{*}$ and outdegree two at every other vertex; let us call such an orientation a $2^{*}$-orientation.

Proposition 3.4 Let $G$ be a plane graph with special vertices $s_{0}$ and $s_{1}$ on the outer face. Weak labelings of $G$ are in bijection to pairs $\left(X, X^{*}\right)$ where $X$ is a 2-orientation of $G$ and $X^{*}$ is a $2^{*}$-orientation of $G_{s}^{*}$.

Proof. The mapping from a weak labeling $\phi$ to a pair $\left(X_{\phi}, X_{\phi}^{*}\right)$ of orientations was given in Observations 3.2 and 3.3 For the converse construction, we introduce an auxiliary graph: The completion $\widetilde{G}$ of $G$ is obtained by superimposing $G$ and $G_{s}^{*}$ such that exactly the primal-dual pairs of edges cross, and this crossing is made a new edge-vertex. For $v, e, f$ the numbers of vertices, edges and faces of $G$, the completion $\widetilde{G}$ of $G$ has $v+e+f+1$ vertices and $4 e$ edges. The faces of $\widetilde{G}$ are (almost) in bijection to the angles of $G$, only the outer face of $\widetilde{G}$ corresponds to two angles, the outer ones of $s_{0}$ and $s_{1}$. In order to remedy this, an exceptional edge $e_{o}$ connecting $o_{0}^{*}$ and $o_{1}^{*}$ can be added.

Given a 2-orientation $X$ of $G$ and a $2^{*}$-orientation $X^{*}$ of $G_{s}^{*}$, we induce an orientation on $\widetilde{G}$ by taking the orientation of an edge $e$ for both of its halfedges, see Figure 10 . We seek for a $0-1$ coloring of the inner faces of $\widetilde{G}$ such that, if $v_{e}$ is an edge-vertex and $a$ an outgoing edge at $v_{e}$, then the color of the two faces incident to $a$ are the same. We model this by calling $a$ an irrelevant edge. Edges that are not irrelevant are relevant. Observe that, from the properties of $X$ and $X^{*}$ and the construction, we obtain:

- There are no relevant edges incident to $s_{0}$ and $s_{1}$.

- There is exactly one relevant edge incident to $o_{0}^{*}$ and $o_{1}^{*}$.

- Apart from these exceptions, every vertex of $\widetilde{G}$ is incident to exactly two relevant edges.

It follows that the relevant edges form a union of disjoint simple cycles and a path from $o_{0}^{*}$ to $o_{1}^{*}$ which, by adding $e_{o}$ as relevant edge is also closed into a cycle. Starting with color 0 in the face containing $s_{0}$, there is a unique extension to a 0-1 coloring of the faces in the graph of relevant edges and hence to a coloring of the faces of $\widetilde{G}$. It is routine to check that this indeed yields a weak labeling and that the two mappings are inverse to each other.

A nice consequence of the above proposition is that it yields a characterization of plane graphs admitting a weak labeling: Given a graph $G=(V, E)$ and a function $\alpha: V \rightarrow \mathbb{N}$, an $\alpha$-orientation is an orientation $X$ of $G$ such that the outdegree of each $v$ is as prescribed by $\alpha$, i.e., it is $\alpha(v)$. It is known that $G$ admits an $\alpha$-orientation if $\sum_{v \in V} \alpha(v)=|E|$ and for all $W \subset V$ the number of edges incident to vertices in $W$ is at least as large as $\alpha(W)=\sum_{v \in W} \alpha(v)$. Moreover, the question whether $G$ admits an $\alpha$-orientation can be translated into a flow-problem [6]. The flow problem can be solved in $O\left(n^{3 / 2}\right)$ with the algorithm of Miller and Naor [14], see also [22]. A polynomial time recognition algorithm for plane graphs admitting 


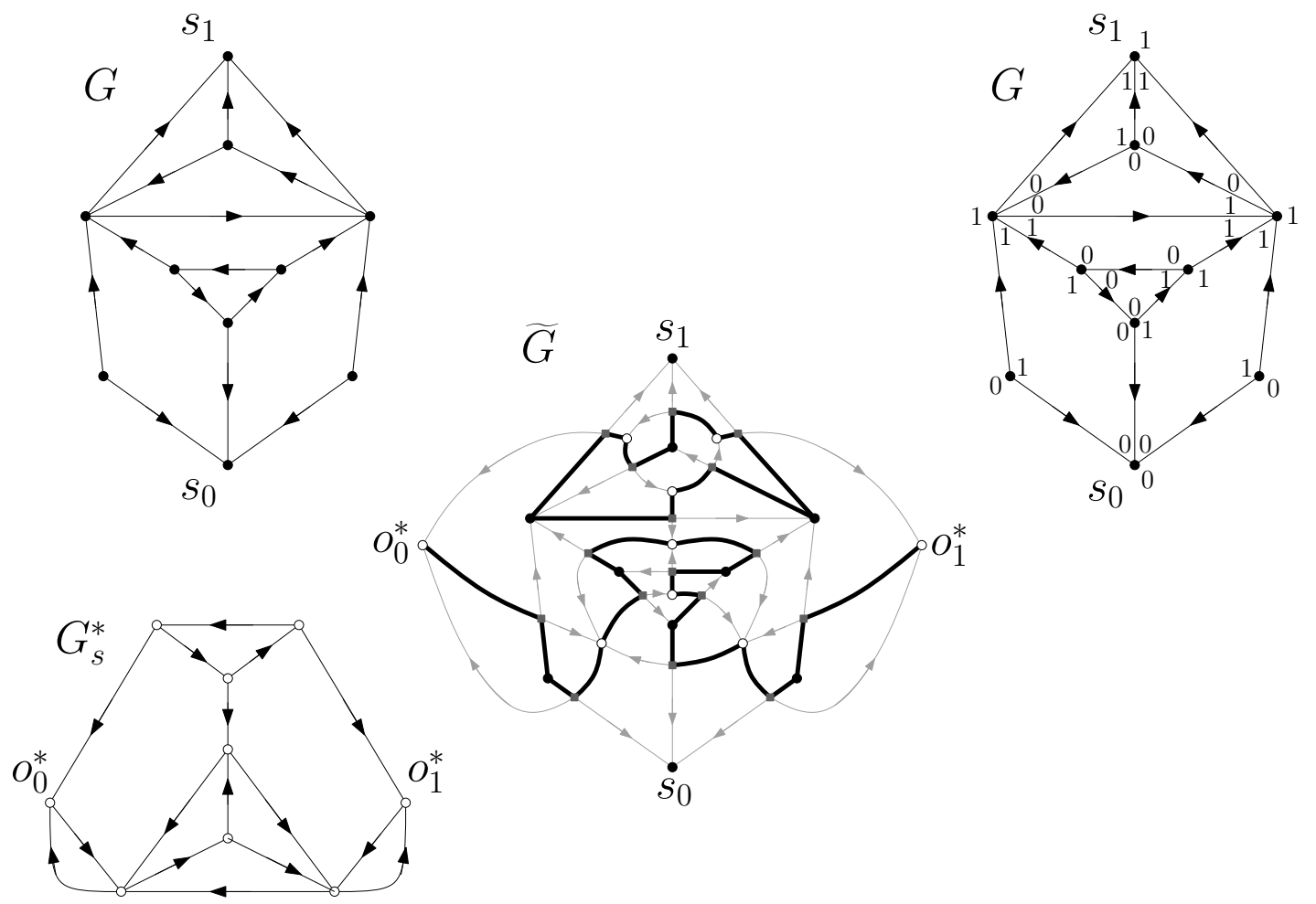

Fig. 10: Orientations $X$ and $X^{*}$, the relevant edges of $\widetilde{G}$ and the resulting weak labeling of $G$.

a weak labeling would first decide whether $G$ and $G_{s}^{*}$ admit a 2- resp. a $2^{*}$-orientation. In the positive case such orientations $X$ and $X^{*}$ could be transformed into a weak labeling of $G$ in linear time. We summarize:

Theorem 3.5 Plane graphs on $n$ vertices admitting a weak labeling can be recognized in $O\left(n^{3 / 2}\right)$.

From the theory of $\alpha$-orientations developed in [6] it also follows that the sets of 2-orientations of $G$ and of $2^{*}$-orientations of $G_{s}^{*}$ carry a natural distributive lattice structure. The product of these two distributive lattices is a distributive lattice on the set of all weak labelings of a plane graph. The ideas on how to define the lattice structure on 2-orientations of a plane graph are explained in Subsection 2.4

\subsection{Schnyder-like properties for weak labelings}

Let $G$ be a plane graph with a weak labeling. As before, we denote by $T_{i}$ the set of oriented edges colored $i$ and by $T_{i}^{-1}$ the set of edges colored $i$ with reversed orientation.

The following proposition is very much like Schnyder's main lemma in [20]. However, in a weak labeling a vertex can have out-degree two in $T_{i}$ wherefore $T_{i}$ need not be a tree, see e.g. Figure 10

Proposition 3.6 If $G$ is a plane graph with a weak labeling, then there is no directed cycle in $T_{0} \cup T_{1}^{-1}$, nor in $T_{1} \cup T_{0}^{-1}$. 
Proof. Suppose that there is a directed cycle $C$ in $T_{0} \cup T_{1}^{-1}$. Clearly we may assume that $C$ is simple, hence, has a well defined interior. Consider $G$ with the original 2-orientation $T_{0} \cup T_{1}$ and define the following counters:

$$
\begin{aligned}
k & =\# \text { vertices on } C . \\
t & =\text { \#vertices in the interior of } C . \\
s & =\text { \#faces in the interior of } C . \\
p & =\text { \# edges pointing in } T_{0} \cup T_{1} \text { from } C \text { into the interior. } \\
q & =\text { \# edges on } C \text { with two different labels on the inner side. }
\end{aligned}
$$

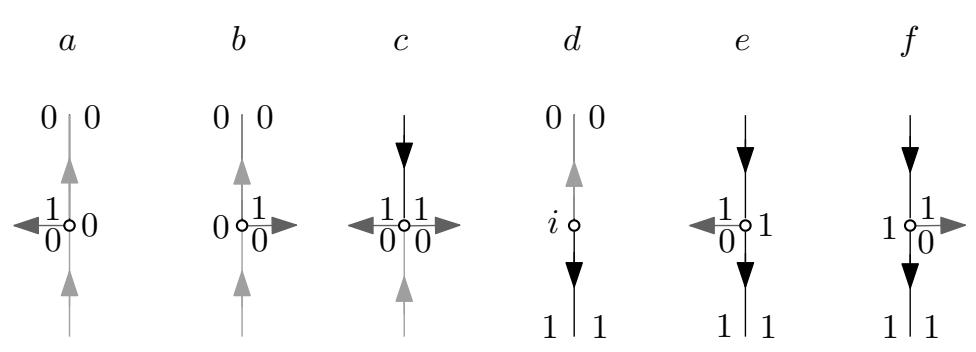

Fig. 11: Vertex types on $C$, where the vertical edges are those on $C$ and the interior of $C$ is assumed to be to the left of them. The schematic drawings show only the edges that are relevant for the counting argument.

Claim A. $p=q$.

Figure 11 shows all types of vertices which can occur on $C$; for type $d$ the label $i$ can be 0 or 1 . Associate each edge that has two different labels on the inner side with its tail-vertex. We find that $q$ equals the number of vertices of types $a, d$ and $e$. The value of $p$ is the number of vertices of types $a, c$ and $e$. Since vertices of type $d$ correspond to a transition from 0 -colored edges to 1-colored edges while vertices of type $c$ correspond to a transition from 1 -colored to 0 -colored edges, it follows that they are equinumerous. This proves the claim.

We now observe that the number $g$ of edges which are on $C$ or in the interior of $C$ can be expressed in several ways:

$$
\begin{aligned}
g & =(k+t)+(s+1)-2 \\
g & =2 t+k+p \\
g & =2 s+k-q
\end{aligned}
$$

Formula (1) is nothing but Euler's formula for the graph restricted to $C$ and its interior. Formula (2) is obtained by counting the out-degrees: Every vertex in the interior of $C$ has out-degree 2 and the sum of all out-degrees of vertices on $C$ is $k+p$. Formula (3) follows from counting changes of labels along edges: By the edge rule (G2) the number of these changes equals the number of edges. By the weak face rule 
$\left(\mathrm{G} 3_{w}\right)$ each of the $s$ faces interior to $C$ contributes two such changes. In addition there are $k-q$ edges on $C$ which have the label change in the outside.

Subtracting (2) and (3) from the double of (1) yields $0=-2-p+q$, which is a contradiction to Claim A.

\section{Generalized strong labelings}

In Section 3 we introduced weak labelings, which can only exist for plane graphs with $n$ vertices and $2 n-4$ edges. In Section 2 we studied strong labelings, i.e., conditions $(\mathrm{G} 0),(\mathrm{G} 1),(\mathrm{G} 2)$ and $(\mathrm{G} 3)$, and saw that the existence of strong labelings characterizes quadrangulations. In this section we modify the edge rule (G2) in order to have similar labelings for a larger class of bipartite plane graphs. The following is inspired by the generalization [5] of Schnyder woods for 3-connected plane graphs. We will always assume that one color class of a bipartite graph has been selected to be the white class, the other one is the black class.

Definition 4.1 A generalized strong labeling for a bipartite plane graph with special vertices $s_{0}$ and $s_{1}$ (of any color) on the outer face is a mapping from its angles to the set $\{0,1\}$ which satisfies:

(G0) Special vertices: All angles incident to $s_{i}$ are labeled $i$.

(G1) Vertex rule: For each vertex $v \notin\left\{s_{0}, s_{1}\right\}$, the incident labels form a non-empty interval of $1 s$ and a non-empty interval of $0 s$.

$\left(\mathrm{G} 2_{g}\right)$ Generalized edge rule: For each edge, the incident labels form one of the six patterns shown in Figure 12.

$\left(\mathrm{G} 3_{g}\right)$ Generalized face rule: Each face has exactly one pair of adjacent 0-labels and one pair of adjacent 1-labels.

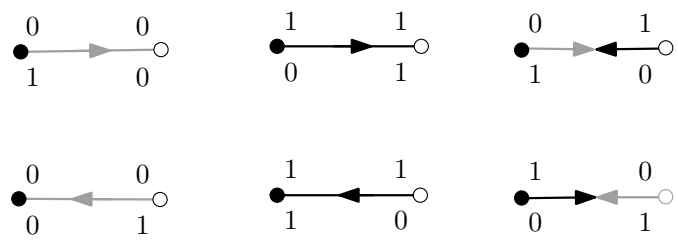

Fig. 12: Generalized edge rule.

Observe that the generalized face rule $\left(\mathrm{G} 3_{g}\right)$ coincides with the face rule $(\mathrm{G} 3)$ for the case of quadrangulations. Figure 17 shows several examples of generalized strong labelings. It should be noted that the degree of the special vertices $s_{0}$ and $s_{1}$ in a graph with such a labeling can be one, e.g., the 2-path $s_{0}-v-s_{1}$ admits a generalized strong labeling.

Another useful fact that can be seen from $(\mathrm{G} 0)$ together with $\left(\mathrm{G} 2_{g}\right)$ is that the edge on the outer face $F_{\text {out }}$ which contains $s_{0}$ and which has $F_{\text {out }}$ to its right when traversed from its white vertex to the black vertex has two adjacent labels 0 in $F_{\text {out }}$.

Lemma 4.2 A generalized strong labeling of a quadrangulation has only edges of the four types of the edge rule (G2), which verify Lemma 2.4, i.e., it has no bidirected edge. 
Proof. A quadrangulation on $n$ vertices has $n-2$ faces and $2 n-4$ edges. Rules (G3 $3_{g}$ ) and (G0) imply that there are at most two color changes in the face walk of each face. In total this yields $2 n-4$ color changes. Since each edge contributes at least one color change there can be no edge contributing two color changes.

Lemma 4.3 A face in a graph equipped with a generalized strong labeling is labeled as shown in Figure 13

Proof. Rule $\left(\mathrm{G} 3_{g}\right)$ implies that in $F$ there is a pair of adjacent 0-labels. Let $u_{0}$ and $u_{1}$ be the vertices of this edge numbered such that in clockwise order $u_{0}$ precedes $u_{1}$. Inspecting Figure 12 i.e., rule $\left(\mathrm{G} 2_{g}\right)$, we find that $u_{0}$ is white and $u_{1}$ is black. Similarly, if $v_{0}$ and $v_{1}$ are the two vertices with adjacent 1-labels in clockwise order then $v_{0}$ is white and $v_{1}$ is black. Between the two special edges there are alternations of labels and colors of some even length on both sides. Note that rule $\left(\mathrm{G} 2_{g}\right)$ allows to identify the complete labeling of edges $\left(u_{2 k}, u_{2 k+1}\right)$ and $\left(v_{2 k}, v_{2 k+1}\right)$ for all $k \geq 1$. They are bidirected.

The generic picture of labels in a face also holds for $F_{\text {out }}$ if the appropriate 'clockwise' order for this face is used, i.e., relative to some point in $F_{\text {out }}$ if $G$ is embedded on the sphere.

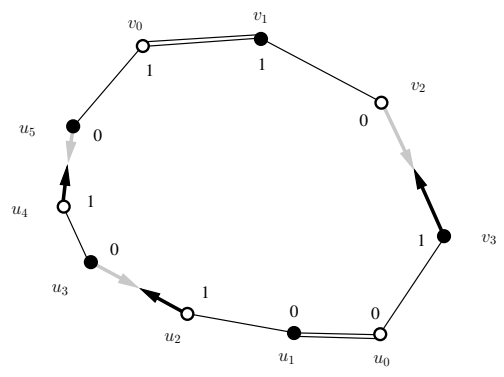

Fig. 13: A generic face in a generalized strong labeling.

Bonichon et al. [3] have introduced operations on Schnyder woods which they call merge and split. A split takes a bidirected edge and makes it unidirected, to account for the reduced out-degree at one of the vertices of the edge a new edge is introduced. A merge is the inverse operation; it takes an angle with two unidirected edges, one of them incoming the other outgoing. The outgoing edge is removed and the incoming edge is made bidirected. We define similar operations for generalized strong labelings. The four possible instances for split and merge are defined by Figure 14, a split is done by replacing a situation from the upper row by the situation below; a merge, conversely, replaces a situation in the lower row by the one above it. Note that a bidirected edge can be split in two ways but the new edge of both splits is introduced in the same face.

Lemma 4.4 If $G$ is a bipartite graph with a generalized strong labeling $B$ and a labeling $B^{\prime}$ of $G^{\prime}$ is obtained from $(G, B)$ by a split or merge, then $B^{\prime}$ is a generalized strong labeling of $G^{\prime}$.

Proof. The labeling $B^{\prime}$ inherits (G0) and (G1) from $B$. All edges in the figure are legal in the sense of $\left(\mathrm{G} 2_{g}\right)$. The least trivial thing is to verify $\left(\mathrm{G} 3_{g}\right)$ for the split. Let us concentrate on the split of the first column where we have given names to the objects. From the generic labeling of a face (Figure 13) it 

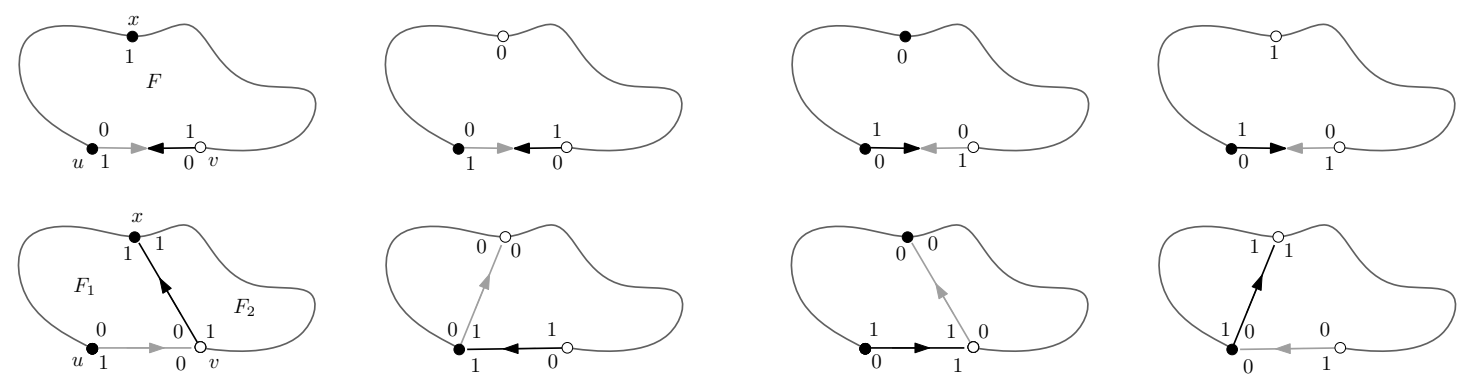

Fig. 14: Split and merge for generalized strong labelings.

follows that $v=u_{2 k}$ and $u=u_{2 k+1}$ for some $k \geq 1$. Since the two black vertices $u$ and $x$ have different labels in $F$ we can conclude that $x=v_{2 l+1}$ for some $l \geq 0$. It is now readily seen that the labels used at the split-edge complete a legal face labeling on either side.

Lemma 4.5 Let $G$ be a bipartite graph with a generalized strong labeling. If $G$ has an inner face which is not a quadrangle, then there is a bidirected edge which can be used in a split.

Proof. If $G$ is not a quadrangulation then there is a face $F$ with at least six edges. The generic labeling of a face (Lemma 4.3 implies that there is a bidirected edge $\left(u_{2 k}, u_{2 k+1}\right)$ or $\left(v_{2 k}, v_{2 k+1}\right)$. From the previous lemma we conclude that such an edge can be split (in two ways) and the resulting labeling is a generalized strong labeling.

If the outer face has more than four edges and the special vertices are of the same color, then again there is a bidirected edge that can be split. If the special vertices are of distinct color, however, it may happen that every split closes a cycle that separates the special vertices, thus violating $(\mathrm{G} 0)$.

Corollary 4.6 If $G$ is a bipartite graph with a generalized strong labeling and the special vertices of $G$ are of the same color, then there is a sequence of edge splittings which lead to a quadrangulation with a strong labeling.

If $e=u v$ is a bidirected edge, then we regard $e$ as outgoing at both vertices $u$ and $v$. With this in mind we conclude from the strong edge rule $\left(\mathrm{G} 2_{g}\right)$ :

Lemma 4.7 The turning rule (Lemma 2.5 and Figure 3) holds for generalized strong labelings.

Given a graph $G$ with a generalized strong labeling, let $T_{0}$ be the set of oriented gray edges and let $T_{1}$ be the set of oriented black edges. Again $T_{i}^{-1}$ is the set of edges of $T_{i}$ with reversed orientation.

Lemma 4.8 $T_{0} \cup T_{1}^{-1}$ and $T_{1} \cup T_{0}^{-1}$ are acyclic. Moreover, $T_{i}, i \in\{0,1\}$, is a directed tree with sink $s_{i}$ that spans all vertices but $s_{1-i}$.

Proof. If the special vertices are of different color add a new special vertex $s_{0}^{*}$ connected to $s_{0}$ with a new edge. The labeling is easily extended as to make $s_{0}^{*}$ and $s_{1}$ the special vertices.

Use edge splits to get to a quadrangulation $Q$. The acyclicity of $T_{0} \cup T_{1}^{-1}$ where $T_{i}$ are the edge sets defined by the orientation of $Q$ was shown in Lemma 2.8 . Note that since a merge has precisely the effect of deleting an edge from $T_{0} \cup T_{1}^{-1}$, this cannot introduce cycles. 
The statement about the trees follows from the acyclicity of $T_{i}$ and the fact that every non-special vertex has outdegree one in $T_{i}$.

The lemma implies that, again, we can define the $i$-path $P_{i}(v)$, of a vertex $v$ as the directed path in $T_{i}$ from $v$ to the sink $s_{i}$. These paths allow, in turn, the definition of the regions $R_{i}(v)$ of a vertex. Consider the numbers $f_{0}(v)$ counting the number of faces in $R_{0}(v)$, i.e., the region to the right of $P_{0}(v)$. These numbers again obey a nice alternation property, namely, if $x y$ is an edge of color 0 with black vertex $x$ and white vertex $y$, then $f_{0}(x) \leq f_{0}(y)$. If the color of $x, y$ is 1 and $x$ is black and $y$ white, then $f_{0}(x) \geq f_{0}(y)$. However, we lose the property that the numbering $f_{0}$ yields a 2-book embedding; this is due to the fact that a 0 -path $P_{0}(u)$ and a 1-path $P_{1}(v)$ can cross by using the two directions of a bidirected edge.

\subsection{Distributive lattice and flips for generalized strong labelings}

In Section 2 we showed that strong labelings for quadrangulations are in bijection to 2-orientations. This allowed us to identify a flip operation on strong labelings which generates a distributive lattice on the set of all strong labelings. The following construction allows to prove equivalent results in the case of generalized strong labelings.

The orientation induced by a generalized strong labeling on $G$ has the somewhat strange property that it may contain bidirected edges. We encode this orientation by a "regular" orientation of a larger graph: Let $G$ be a connected bipartite plane graph with distinguished color classes black and white. Define a graph $S_{G}$ as follows: As vertices of $S_{G}$ take the union of the vertices, edges and faces of $G$. Every edge-vertex has degree three and is connected to the two endpoints and to the face on its right when traversed from white to black. Figure 15 shows an example. The construction is inspired by the completion of a plane graph as used in the proof of Proposition 3.4. Similar constructions have been considered in the context of Schnyder woods, see e.g. [6].
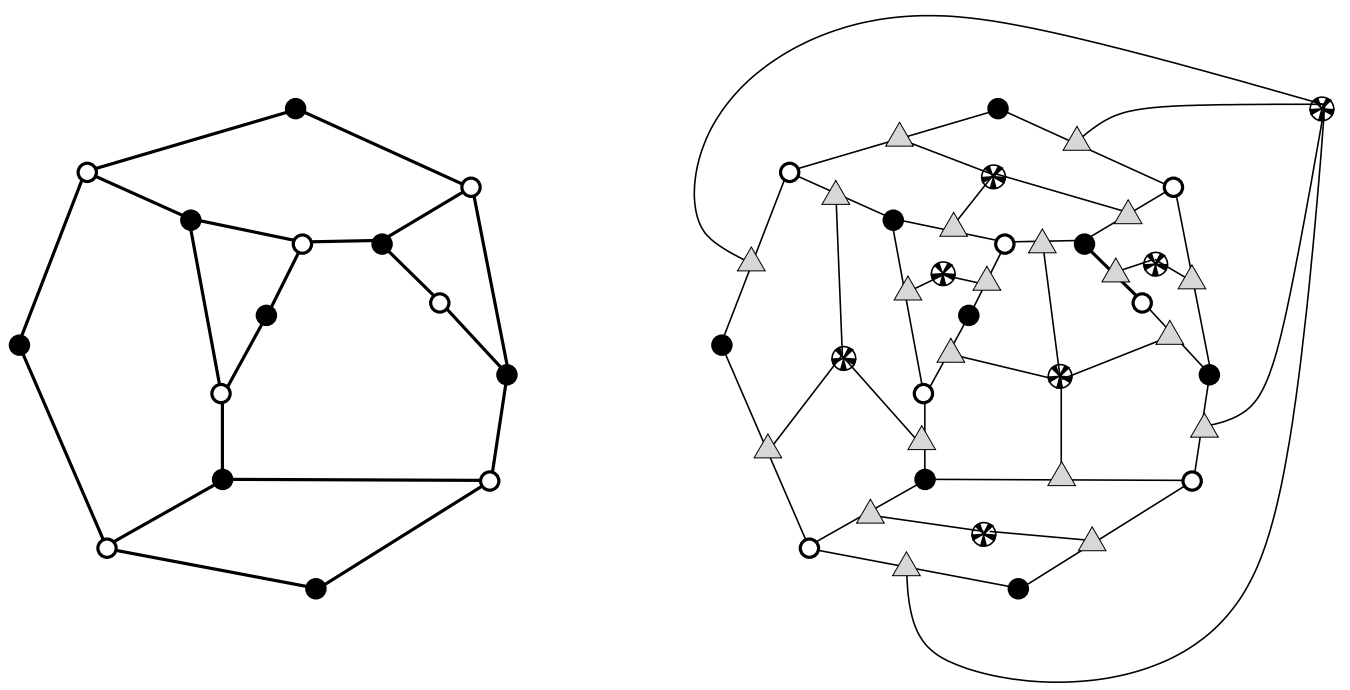

Fig. 15: A graph $G$ (left) and the corresponding $S_{G}$ (right). 
Proposition 4.9 Generalized strong labelings of $G$ with $s_{0}$ and $s_{1}$ on the outer face are in bijection with orientations of $S_{G}$ which have the following outdegrees

$$
\operatorname{outdeg}(x)= \begin{cases}0 & \text { if } x \in\left\{s_{0}, s_{1}\right\}, \\ 1 & \text { if } x \text { is an edge-vertex } \\ 2 & \text { otherwise. }\end{cases}
$$

Proof. Figure 16 shows how to translate from a generalized strong labeling of $G$ to an orientation of $S_{G}$. There is a clear correspondence between the rules (G0) and (G1) and the prescribed outdegrees of original

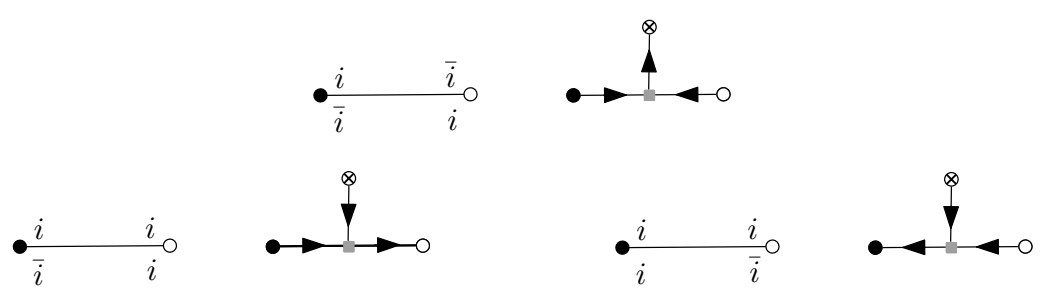

Fig. 16: Translating orientations from $G$ to $S_{G}$ and back. $\bar{i}$ denotes the label $1-i$.

vertices. The generalized edge rule and outdegree 1 for edge-vertices are both assumed for the translation. From the face rule $\left(\mathrm{G} 3_{g}\right)$ together with the restriction of the labeling at edges $\left(\mathrm{G} 2_{g}\right)$ we conclude that face-vertices have outdegree 2 . Note that this also holds for the outer face, the two edges on the outer face which should have repeated labels to satisfy $\left(\mathrm{G} 3_{g}\right)$ connect to the vertices $s_{0}$ and $s_{1}$ which have prescribed outdegree 0 . Therefore, these two edge-vertices receive the two outgoing edges of the vertex of the outer face.

Conversely, if an orientation of $S_{G}$ with the prescribed outdegrees is given we can recover the labeling: start at $s_{0}$ where the labels are known and extend the labeling along edges via the scheme given in Figure 16 .

The orientations of $S_{G}$ described in the proposition are $\alpha$-orientations in the sense of [6]. Hence, the set of all generalized strong labelings of $G$ can be ordered as a distributive lattice. In particular the generalized strong labelings are again flip-connected, where a flip is defined as the complementation of all labels inside a cycle $C$ which is directed in the corresponding orientation of $S_{G}$.

Recall from the discussion preceeding Theorem 3.5 that for given $G$ and a fixed function $\alpha$, the existence of an $\alpha$-orientation can be decided in polynomial time. Together with the proposition, this yields:

Theorem 4.10 Plane graphs admitting a generalized strong labeling can be recognized in polynomial time.

\subsection{Graphs admitting a generalized strong labeling}

So far we have shown that generalized strong labelings have a nice structure. The correspondence with orientations of $S_{G}$ yields polynomial time recognition and an implicit characterization via the criterion for $\alpha$-orientations given on page 126 In this subsection we provide an explicit characterization. 



Fig. 17: Examples of generalized strong labelings.

To introduce into the topic we have two figures. Figure 17 shows some examples of graphs with generalized strong labelings. The four examples on the left illustrate how the colors of the special vertices influence the labeling along the outer face. The generalized strong labelings in these cases are unique. The generalized strong labeling of the larger graph on the right is not unique, e.g., exchanging the two underlined labels leads to another generalized strong labeling. Figure 18 shows some graphs which do

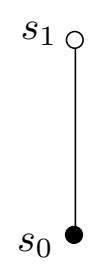

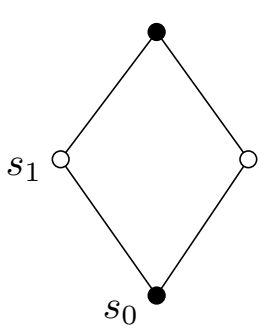
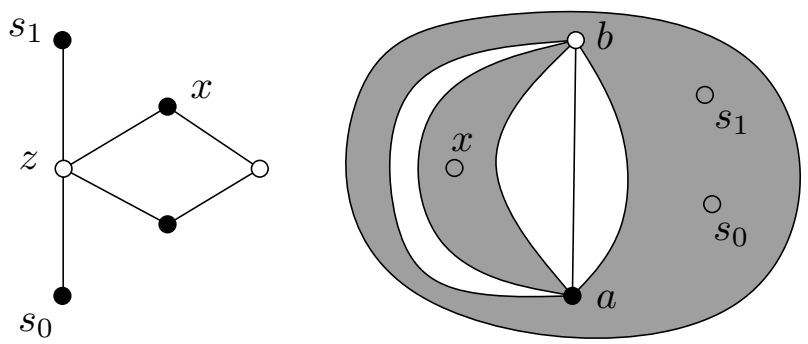

Fig. 18: Some graphs which do not admit a generalized strong labeling.

not admit generalized strong labelings. The first two examples fail to admit a generalized strong labeling simply because their two special vertices are adjacent. Rule (G0) would force the connecting edge to have two identical labels on both of its vertices, which is infeasible by the edge rule. In the middle example there is a cut vertex between $x$ and the two special vertices. The two paths $P_{0}(x)$ and $P_{1}(x)$ would both 
contain $z$ which forces a cycle in $T_{0} \cup T_{1}^{-1}$, which is impossible by Lemma 4.8

An undirected graph with special vertices $s_{0}$ and $s_{1}$ is called weakly 2-connected if it is 2-connected or adding an edge $s_{0} s_{1}$ makes it 2-connected. This is equivalent to saying that every vertex $x$ has a pair of vertex-disjoint paths one leading to $s_{0}$ and the other to $s_{1}$. From the above it follows that being weakly 2-connected is a necessary condition for admitting a generalized strong labeling.

Now consider the sketch on the right of Figure 18. It illustrates the following situation: There is an edge $a b$, vertex $a$ is black and vertex $b$ white. Removing $a$ and $b$ we disconnect a component $C$ with $x \in C$ from the special vertices $s_{0}$ and $s_{1}$. Moreover, component $C$ is to the left of $a b$. If a graph contains such an edge we say that it contains a block with a right chord. Suppose that a graph containing a block with a right chord admits a generalized strong labeling. Disjointness forces the two paths $P_{0}(x)$ and $P_{1}(x)$ to leave the component $C$ through vertices $a$ and $b$. Now consider the orientation of the edge $a b$, if it is directed from $b$ to $a$, then the turning rule for white vertices makes the path $P_{i}(x)$ leaving at $b$ continue through $a$ where the two paths meet, contradiction. If the direction of $a b$ is from $a$ to $b$, then it is the turning rule for the black vertex $a$ which leads to the same kind of contradiction.

With the three cases we have identified all the obstructions against admitting a generalized strong labeling:

Theorem 4.11 Let $G$ be a bipartite plane graph with color classes black and white and two special vertices $s_{0}, s_{1}$ on the outer face. G admits a generalized strong labeling if and only if the following conditions are satisfied.

(1) $s_{0}$ and $s_{1}$ are nonadjacent,

(2) G is weakly 2-connected,

(3) G contains no block with a right chord.

Proof. The "only if" part comes from the above discussion. The proof for the "if" part is by induction on the number of edges. Let $G$ be a graph satisfying the conditions. We concentrate on the case where $s_{0}$ is a black vertex.

Let $e=s_{0} v$ be the first edge in clockwise order which is incident to $s_{0}$ and belongs to the boundary of the outer face (in Figure 17 it is the leftmost edge at $s_{0}$ ). Rule $\left(\mathrm{G} 2_{g}\right)$ implies that $e$ has the duplicate label 0 on the outer face. Now, remove $e$ from $G$ and let $G^{\prime}$ be the resulting graph. There are several cases. The easy cases, discussed first, are considered in Figure 19

The first case is that $G^{\prime}$ satisfies the conditions. Induction implies a generalized strong labeling for $G^{\prime}$. Consider the edge $u v$ on the boundary of the outer face of $G^{\prime}$ which is interior in $G$. In the labeling of $G^{\prime}$ on the outer face the black vertex $u$ has label 1 and the white vertex $v$ has label 0 . The generalized edge rule $\left(\mathrm{G} 2_{g}\right)$ implies that the labels on the opposite side of this edge are inverse, 0 at $u$ and 1 at $v$. Therefore, it is consistent with edge and vertex rules to label the angle between $e$ and $u v$ with 1 and the outer angle of $e$ at $v$ with 0 . This yields a generalized strong labeling of $G$.

Now suppose that $G^{\prime}$ does not satisfy the conditions because condition (2) fails. If $G^{\prime}$ is not connected it has $s_{0}$ as an isolated vertex. Choose $v$ as the special vertex $s_{0}^{\prime}$ for the component of $G^{\prime}$ which contains $s_{1}$. If this component admits a generalized strong labeling we can extend this to the full graph. Otherwise, condition (1) is not satisfied. Hence either the component is just the single edge $s_{0}^{\prime} s_{1}$ or this edge is a left chord to a block which satisfies all three conditions. In both cases it is easy to get to a generalized strong labeling of $G$. 

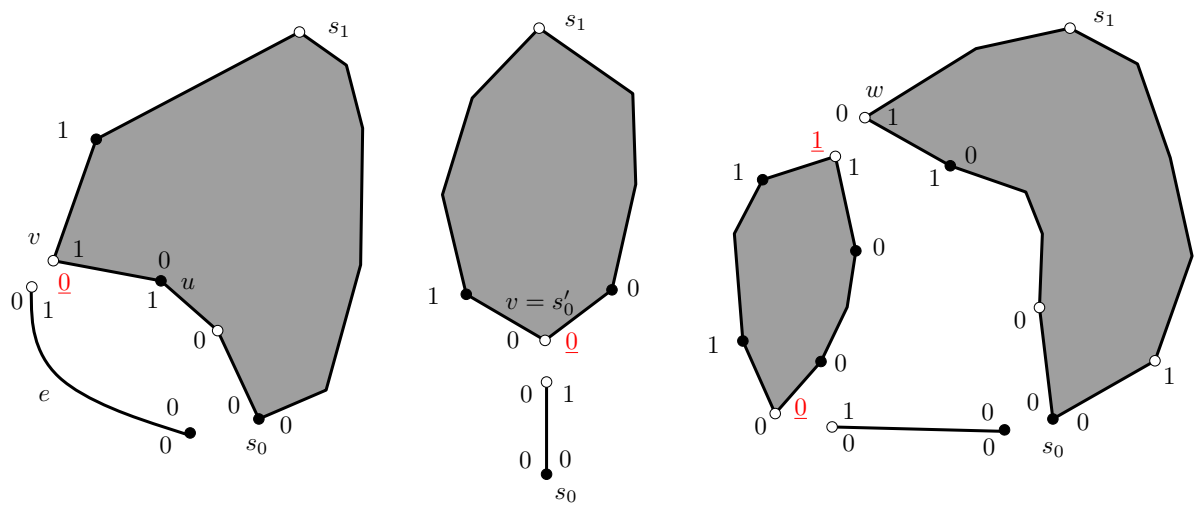

Fig. 19: Constructing the generalized strong labeling in the inductive proof. Underlined labels are inverted in the labeling of $G$.

If $G^{\prime}$ is connected but fails to satisfy (2), then it has a cut vertex. Let $w$ be the cut vertex such that one of the components is weakly 2-connected between $v$ and $w$ and the other is weakly 2-connected between $s_{0}$ and $s_{1}$. The first of these components is either a single edge or it satisfies the conditions. The second component also satisfies the conditions. By induction both components have generalized strong labelings. Again it is straightforward to define a generalized strong labeling based on the generalized strong labelings of the components. The right part of Figure 19 shows the case where $w$ is a white vertex.

It remains to consider the case where condition (3) does not hold for $G^{\prime}$, Figure 20 illustrates the situation. Removing edge $e=s_{0} v$ generates a new block $B$ with a right chord $u w$. Split the graph into two parts. One part consists of block $B$ together with the edge $u w$, let $v=s_{0}^{\prime}$ and $w=s_{1}^{\prime}$ be the special vertices for this part. This part of the graph fulfills the condition so that by induction a generalized strong labeling exists. The other part also has a generalized strong labeling. The left part of the figure shows some of the labels that can be derived by applying Lemma 4.3 to the outer faces. The right part of the figure shows how to recombine them to form a generalized strong labeling of the full graph.

\section{Acknowledgements}

We thank the referees for many hints that helped in improving the presentation.

Apart from the authors' universities, parts of this work were done during the III Taller de Geometría Computacional, organized by the Universidad de Valladolid, and during a visit to the Centre de Recerca Matemàtica.

\section{References}

[1] O. Aichholzer, F. Aurenhammer, P. Gonzalez-Nava, T. Hackl, C. Huemer, F. Hurtado, H. Krasser, S. Ray, B. Vogtenhuber. Matching edges and faces in polygonal partitions. Computational Geometry: Theory and Applications 39: 134-141, 2008.

[2] E. Brehm. 3-orientations and Schnyder 3-tree-decompositions. Diplomarbeit, Freie Universität Berlin, Germany, 2000. 

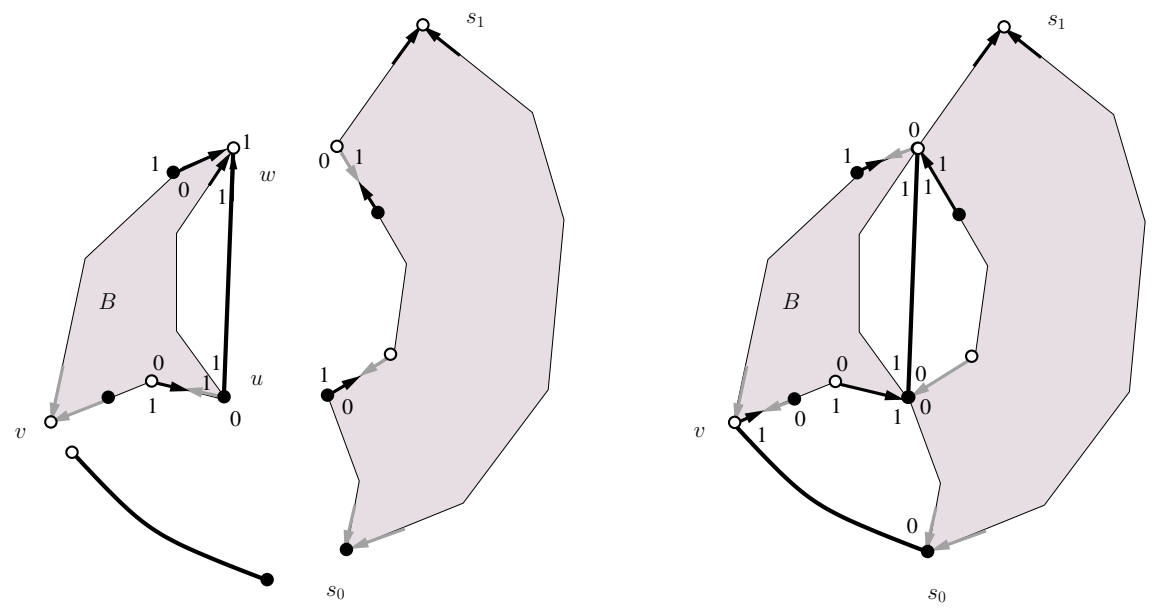

Fig. 20: How to deal with the case when removing $e$ results in a block with a right chord.

[3] N. Bonichon, S. Felsner, M. Mosbah. Convex drawings of 3-connected planar graphs. Algorithmica 47: 399-420, 2007.

[4] V. Dujmovic, D.R. Wood. On linear layouts of graphs. Discrete Mathematics and Theoretical Computer Science 6: 339-358, 2004.

[5] S. Felsner. Convex Drawings of Planar Graphs and the Order Dimension of 3-Polytopes. Order 18: 19-37, 2001.

[6] S. Felsner. Lattice Structure from Planar Graphs. Electronic Journal of Combinatorics 11: 1R15, 2004.

[7] S. Felsner, É. Fusy, M. Noy, D. Orden Bijections for Baxter Families and Related Objects. Journal of Combinatorial Theory, Series A Article in Press, 2010. DOI:10.1016/ j. jcta.2010.03.017

[8] H. de Fraysseix, P. Ossona de Mendez. On topological aspects of orientations. Discrete Mathematics 229: 57-72, 2001.

[9] H. de Fraysseix, P. Ossona de Mendez, J. Pach. A left-first search algorithm for planar graphs. Discrete Computational Geometry 13: 459-468, 1995.

[10] H. de Fraysseix, P. Ossona de Mendez, P. Rosenstiehl. Bipolar orientations revisited. Discrete Applied Mathematics 56: 157-179, 1995.

[11] I. M. Gelfand, M. I. Graev, A. Postnikov. Combinatorics of hypergeometric functions associated with positive roots. In V. I. Arnold et al. (ed.) The Arnold-Gelfand Mathematical Seminars: Geometry and Singularity Theory, 205-221, Boston, 1997.

[12] C. Huemer, S. Kappes. A binary labelling for plane Laman graphs and quadrangulations. 22nd European Workshop on Computational Geometry, pages 83-86, Delphi, Greece, 2006. 
[13] A. S. Lladó, S. C. López Masip. Decompositions of graphs with a given tree. In Actas de las III Jornadas de Matemática Discreta y Algorítmica, 204-211, Sevilla, Spain, 2002.

[14] G. L. Miller and J. Naor. Flow in planar graphs with multiple sources and sinks. SIAM Journal on Computing 24: 1002-1017, 1995.

[15] A. Nakamoto, M. Watanabe. Cycle reversals in oriented plane quadrangulations and orthogonal plane partitions. Journal of Geometry 68: 200-208, 2000.

[16] P. Ossona de Mendez. Orientations bipolaires. Ph.D. thesis, Ecole des Hautes Etudes en Sciences Sociales, Paris, France, 1994.

[17] V. Petrović. Decomposition of some planar graphs into trees. Discrete Mathematics 150: 449-451, 1996.

[18] G. Ringel. Two Trees in Maximal Planar Bipartite Graphs. Journal of Graph Theory 17: 755-758, 1993.

[19] G. Rote, F. Santos, I. Streinu. Expansive motions and the polytope of pointed pseudo-triangulations. In Discrete and Computational Geometry-The Goodman-Pollack Festschrift, 699-736. Algorithms and Combinatorics, vol. 25. Springer 2003.

[20] W. Schnyder. Planar graphs and poset dimension. Order 5: 323-343, 1989.

[21] W. Schnyder. Embedding planar graphs on the grid. In Proceedings of the 1st ACM-SIAM Symposium on Discrete Algorithms 138-148, 1990.

[22] T. Ueckerdt. Complexity of Orientations. in preparation. 